Key points

- Interface selection is mainly influenced by the patient's face shape, mouth/nose breathing pattern, nasal pathology, experience of the staff, patient choice and equipment availability. Interfaces covering the mouth and nose are usually the first choice for acute NIV.

- Compatibility of the chosen interface with the ventilator circuitry should be checked and the exhalation port identified before NIV is started.

- Large air leaks affect the efficacy of NIV and should be avoided, whereas small air leaks can be compensated for by ventilators designed for NIV and are usually tolerated if they do not irritate the patient or disturb sleep.

- Routine assessment of the skin and risk of pressure ulcers, regular pressure relief and skin-protective strategies should be included in the routine application of NIV to reduce discomfort and the occurrence of soft tissue damage.

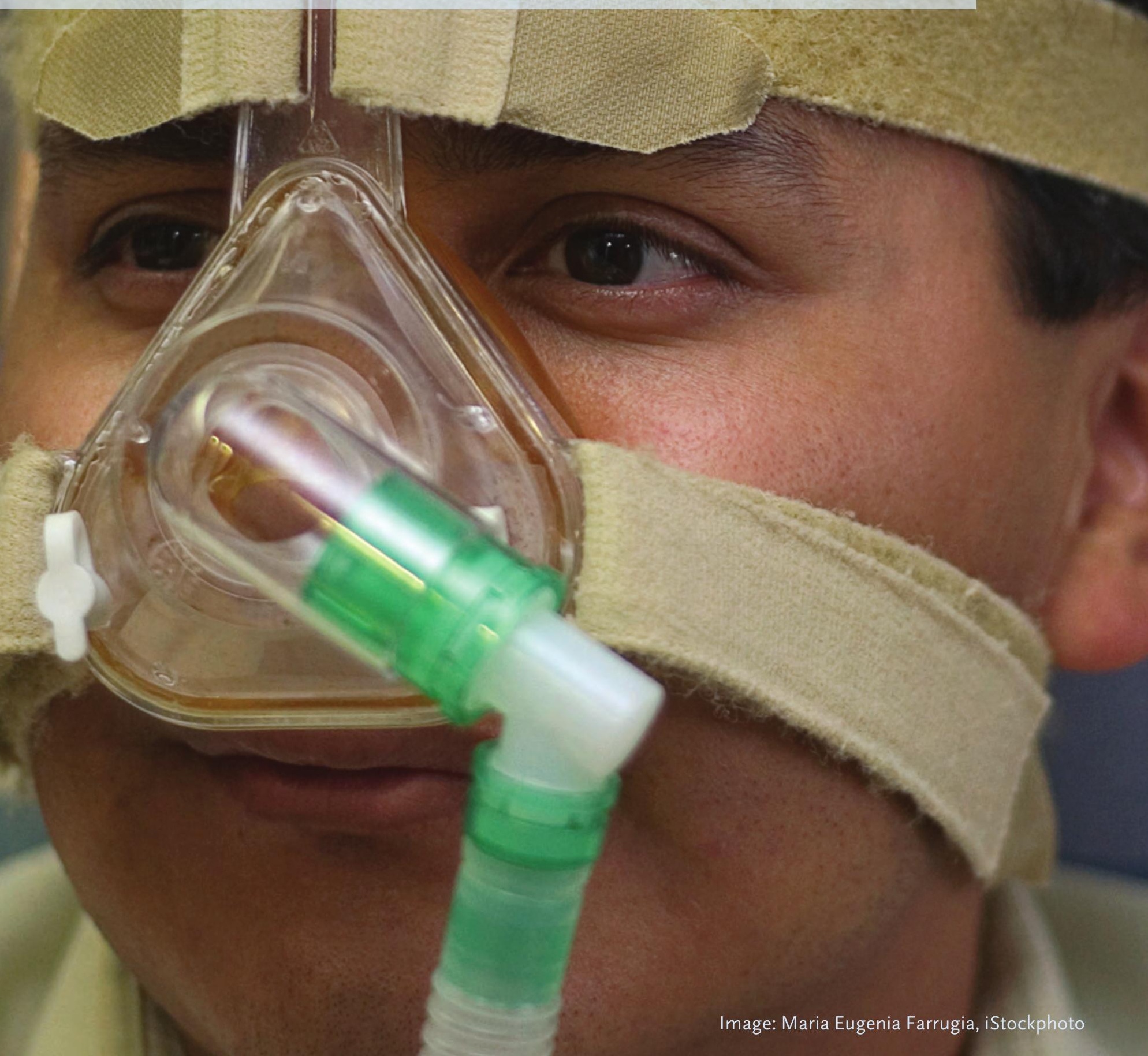




$\begin{array}{llll}\sum_{\text {Anne-Kathrin Brill }} & \text { Dept of Sleep and } & \text { Dept of Sleep and } \\ & \begin{array}{l}\text { Ventilation, Royal Brompton } \\ \text { and Harefield NHS }\end{array} & \begin{array}{l}\text { Ventilation, Royal Brompton } \\ \text { and Harefield NHS }\end{array} \\ & \begin{array}{ll}\text { Foundation Trust, London, } \\ \text { UK }\end{array} & \text { Foundation Trust, Sydney } \\ & \text { Street, London, SW3 6NP, } \\ & \text { UK }\end{array}$

\section{How to avoid interface problems in acute noninvasive ventilation}

\section{Summary}

Noninvasive ventilation (NIV) applied via different interfaces is increasingly used in the treatment of acute respiratory failure. One of the key factors determining the success of NIV is the choice of interface. Interface selection, fitting and handling can be challenging as NIV application can be complicated by discomfort, air leaks, skin damage or conjunctivitis. The aim of this article is to provide practical information on interface choice, technical aspects of mask fitting and prevention of mask-related problems during the acute delivery of NIV.

\section{Educational aims}

- To help the reader to recognise the different features of interfaces and to discuss common interface-related problems

- To provide practical recommendations to facilitate the choice and application of interfaces in order to reduce complications

The use of noninvasive ventilation (NIV) has grown continuously over the past two decades. It has become a standard of care in the treatment of many forms of acute respiratory failure (ARF) and is now frequently applied in intensive care units (ICUs), emergency departments, post-surgical recovery rooms, step-down units and respiratory wards [1-4].
Despite the overall success of the treatment modality, NIV failure rates still range between $18 \%$ and $40 \%$ in the acute setting $[1,3,5-7]$. Primary failure of NIV reflects failure of uptake of the technique and control of respiratory failure; secondary failure is seen when respiratory failure progresses despite optimal NIV use.
Statement of Interest None declared.

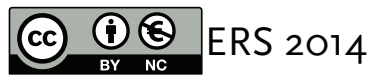

HERMES syllabus link: module E.1.6 
Patient selection, underlying pathology, the severity of ARF, expertise with NIV and interface tolerance all have a major impact on the success or failure of NIV. Interface-related problems such as air leaks, skin lesions or discomfort are still common reasons for poor adaptation to NIV and NIV failure [8-10]. They can often be addressed successfully with basic knowledge of the principles of mask fitting and handling.

The purpose of this article is to provide an overview of strategies to anticipate, prevent and manage interface-related problems in acute NIV.

\section{Interface selection}

A greater variety of different interface types, styles and materials have been developed by device manufacturers in recent years. This allows a suitable interface to be found for nearly every patient in most situations, but having to deal with many different interfaces and be able to select the most appropriate one can be challenging for clinical staff. There are six main classes of interfaces that are commercially available in various sizes, all of which have their advantages and disadvantages (table 1). Custom made masks are another option, but in the acute setting with critically ill patients, there is usually not enough time to fabricate these.

Interface selection is mainly influenced by the patient's individual characteristics (i.e. facial anatomy, breathing pattern and individual level of comfort) and clinical effectiveness, but the experience of the staff, equipment availability and economic aspects are also relevant. In the few studies comparing different types of interfaces in acute NIV, the improvements in respiratory parameters, i.e. dyspnoea, respiratory rate and arterial blood gases, were usually similar and there is no strong scientific evidence that one type of mask is necessarily or consistently better than others in terms of clinical efficiency. However, patients in ARF often mouth-breathe and, even if they can be started on a nasal mask, a switch to an alternative interface covering the nose and mouth is necessary in many patients if mouth air leaks occur [11, 12]. In clinical practice the most common initial interface to treat ARF with NIV is, therefore, an oronasal mask. This was reflected in the results of a large web-based survey in Europe and North America, with oronasal masks (70\%) being the first choice followed by total face masks, nasal masks and helmets [13].

\begin{tabular}{|c|c|c|c|c|c|c|}
\hline & $\begin{array}{c}\text { Oronasal } \\
\text { mask }\end{array}$ & $\begin{array}{l}\text { Total face } \\
\text { mask }\end{array}$ & Helmet & $\begin{array}{l}\text { Nasal } \\
\text { mask }\end{array}$ & $\begin{array}{l}\text { Nasal } \\
\text { prongs }\end{array}$ & $\begin{array}{l}\text { Mouth } \\
\text { pieces }\end{array}$ \\
\hline Acute setting & • & $\bullet$ & $\bullet$ & 0 & O & O \\
\hline Use outside HDU/ICU & $\bullet$ & • & & $\bullet$ & $\bullet$ & • \\
\hline Less claustrophobic & & 0 & O & $\bullet$ & $\bullet$ & $\bullet$ \\
\hline $\begin{array}{l}\text { More likely to have leaks } \\
\text { in the acute setting }\end{array}$ & $\bullet$ & & & $\bullet$ & $\bullet$ & $\bullet$ \\
\hline Nasal patency required & & & & $\bullet$ & $\bullet$ & \\
\hline $\begin{array}{l}\text { Coughing and } \\
\text { expectoration is easier }\end{array}$ & & & & $\bullet$ & - & \\
\hline $\begin{array}{l}\text { Useful for prominent } \\
\text { facial anatomy }\end{array}$ & & $\bullet$ & $\bullet$ & & - & • \\
\hline High level of noise & & & • & & & \\
\hline $\begin{array}{l}\text { No pressure on the } \\
\text { nasal bridge }\end{array}$ & & $\bullet$ & $\bullet$ & & $\bullet$ & $\bullet$ \\
\hline High gas flow required & & & $\bullet$ & & & \\
\hline Chance of eye irritation & $\bullet$ & ○ & O & $\bullet$ & & \\
\hline Speaking is easier & & & - & - & $\bullet$ & \\
\hline
\end{tabular}

Table 1

Different interface types. HDU: high dependency unit. Closed circles: applicable to the interface; open circles: an alternative, but less common or less frequent option. 
The ability to try different mask types and sizes quickly may enhance tolerance as patients who do not adapt well to one type of mask may comply with another. Having a number of different mask types and sizes attached to the NIV ventilator can, therefore, be advantageous, although financial constraints may limit those available. Most masks come with fitting gauges to avoid selection of a mask that is too large or too small and clinicians are encouraged to use them to reduce complications. The mask should not encroach on the corners of the eyes or lips. Changing the interface can reduce NIV failure, and is always worth trying if large leaks are seen and demonstrated on ventilator software or the type of interface is not well tolerated by the patient, but experimenting with interfaces should not delay intubation in a severely ill patient if NIV is clearly failing. When switching to a different mask, trigger sensitivity, pressurisation level, and compatibility with the circuitry must be checked.

In the long-term setting, nasal masks are rated to be more comfortable than other mask types [14] and cause skin problems less often. If the patient is stable and there are no major mouth air leaks, it may be reasonable to swap to a nasal mask or nasal prongs. In addition, patients in the post-acute setting can sometimes be encouraged to continue NIV by switching to a less claustrophobic interface type.

\section{Carbon dioxide rebreathing: dead space and ventilator circuits}

Depending on the ventilator and circuit used, NIV can be applied either via an open singlelimb circuit or a closed double-limb circuit. An open single-limb circuit requires a vented mask with a built-in exhalation port or a nonvented mask and an additional exhalation valve in the circuit to allow carbon dioxide $\left(\mathrm{CO}_{2}\right)$ removal. A closed double-limb circuit is used with a nonvented mask and has an exhalation port or filter for $\mathrm{CO}_{2}$ removal within the system. It is essential to be aware of which circuit is used when the mask is chosen. The wrong combination, for example a nonvented mask in an open single-limb system and no exhalation port in the system, can be fatal. Also, an exhalation port should never be obstructed intentionally (e.g. taping up the holes in the mask) to reduce leakage. Different colours on the mask or the mask elbow, with a blue colour representing nonvented masks and a clear entrainment elbow for vented models (fig. 1), can give guidance, but it is not always a guarantee. For safe treatment, it is important to locate the exhalation port, assure its patency and check compatibility of the circuit and interface prior to the start of NIV.

The interface itself adds an additional dead space to the system, which can increase $\mathrm{CO}_{2}$ rebreathing. Bench studies have suggested that $\mathrm{CO}_{2}$ rebreathing increases with interfaces that have a higher internal volume and decreases with a built-in exhalation port [15]. The in vivo effect is only small and, with the exception of the helmet, facial masks are usually interchangeable [16].

\section{Initiation of NIV}

The first few hours of acute NIV are extremely important, and time spent fitting the mask and building the patient's confidence is well invested. Having a mask strapped immediately over the face can be very frightening for a breathless patient in ARF, particularly if the person is naïve to NIV. A careful explanation of what will happen and why NIV is used as well as a description of the sensation the

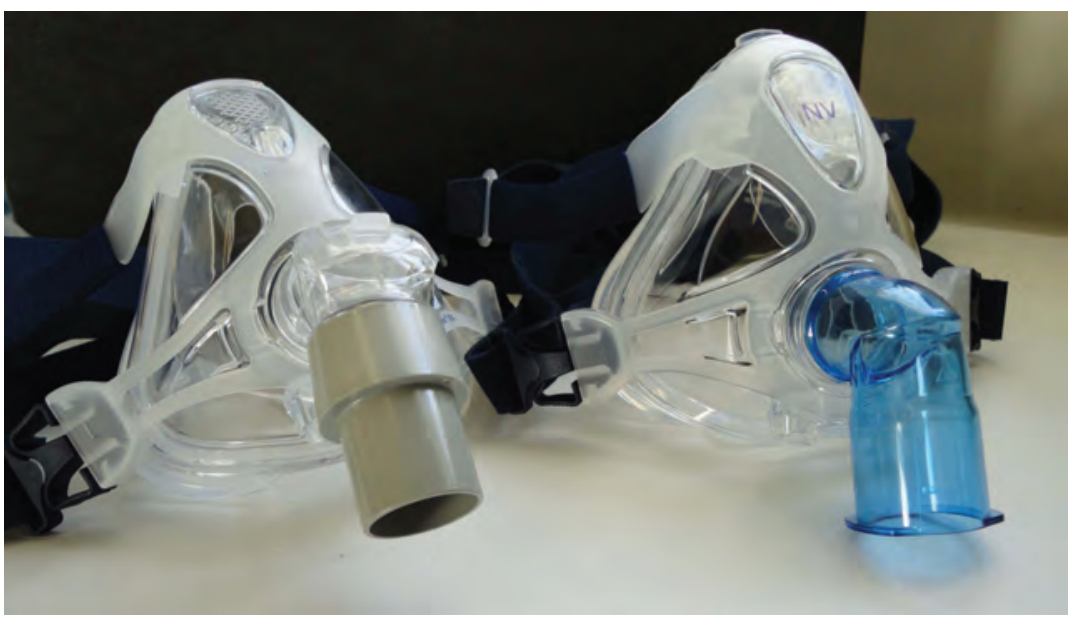

Figure 1

A vented mask with a clear elbow, grey adapter and integrated exhalation port on the left for single limb circuits. A nonvented mask with a blue elbow for dual limb systems is shown on the right. Masks manufactured by ResMed Ltd. (Abingdon, UK). 
patient is likely to experience can help to facilitate the treatment. Initiating NIV with lower pressures while holding the mask or having the patient hold the mask in place and not attaching the straps until the patient is ready to tolerate it can also help the patient to acclimatise. Once the patient is able to tolerate the mask, it can be secured with the head straps.

\section{Prevention and management of mask- related side-effects}

At this point, the clinician who fits the mask has to achieve a good mask seal while providing the maximal possible comfort and preventing mask-related complications. Initially reducing air leaks is the major goal of the mask fitting, but the longer the treatment lasts, the more likely it is that complications, including facial skin erythema, skin breakdown, rash, conjunctivitis or dryness of the mucosa, occur.

\section{Air leaks}

Intentional air leaks are incorporated into the design of most NIV circuits and are necessary for $\mathrm{CO}_{2}$ removal, but unintentional leaks around the edge of the interface or through the mouth can cause problems. Large air leaks interfere with the effectiveness of the treatment, while small air leaks can irritate the patient, cause conjunctivitis or create noise.

To provide adequate ventilation during treatment with NIV, it is important to maintain the pressure gradient from the ventilator through the tubing, and from the mask to the patient's pharynx and subsequent airways. Large air leaks can cause a significant drop in the delivered intra-alveolar pressure, reduce tidal volume and lead to patient-ventilator asynchrony by affecting trigger functions. All this contributes to ineffective treatment, which is associated with NIV failure [17]. Therefore, large air leaks have to be minimised during NIV. Ventilators designed for NIV can compensate for smaller air leaks to a variable extent [18] and a small amount of air leakage can be accepted as long as the leak does not disturb the patient.
Besides using an appropriate interface type, with the correct size and headgear, there are several other interventions to reduce air leaks. The most obvious, but least comfortable, solution seems to be further tightening of the head straps. Sometimes, loosening the head straps or temporarily lifting the mask from the face and replacing it can have an equivalent or better effect without causing more pressure on the patient's face. For example, masks that incorporate double spring air-filled cushions will fit and seal better if the cushions are filled with air and not tightly pressed against the face. Different types of mask cushions, for example hydrogel or foam cushions, are also available and changing the cushion can sometimes improve mask fit. In addition, mask support rings, thin silicone or cotton comfort flaps, mask liners, hydrogen or foam seals can be tried. If the patient's chin keeps dropping, masks with a special under-chin support are a useful alternative option.

With the use of nasal masks or nasal prongs, air leaks through the mouth can become problematic. Although the use of chinstraps or mouth taping have been suggested, changing to a mask type that covers mouth and nose is usually the recommended strategy in ARF. Chinstraps can successfully reduce air leaks via the mouth in selected patients in the chronic setting [19], but they are not always tolerated and there is little evidence to support their use in ARF. Also, chinstraps can cause discomfort, jaw or tooth pain and their use should not be used to prolong an ineffective treatment. Mouth taping has been shown to decrease air leak from the mouth in a physiological study in the chronic setting [20], but it cannot be recommended in ARF for obvious safety reasons.

If a disturbing air leak cannot be controlled by other means and a change to a different interface type is not possible, a small reduction of peak inspiratory pressure or switching from a volume-controlled to a pressure-targeted mode of ventilation can be tried. Conversely, increasing the pressure with pressure-targeted ventilation or tidal volume with volume-targeted ventilation can improve minute ventilation despite the higher leakage [21], as long as the patient tolerates such a strategy. The additional volume will add to the leakage, but nevertheless can increase minute ventilation. 


\section{Eye irritation}

Smaller air leaks near the eyes can cause irritation or conjunctivitis if they are not noticed. Assessing for small air leaks by placing the back of the hand over the area, and asking the patient about eye irritation routinely throughout the treatment and every time the mask is fitted, can help to prevent this complication. Artificial tears can be applied if the eyes are affected.

\section{Noise}

If air leaks create noise, the mask should be refitted and a lining can be added to seal it. There is no problem with air leaks with the helmet interface, but the high-flow system creates a higher level of noise within the device than other interfaces and providing ear plugs for the patients can be helpful.

\section{Mask pressure}

A certain amount of pressure is usually needed to keep the NIV mask in place and seal it, but an excessively tight fit is unnecessary. Too much pressure on the face causes discomfort, contributes to skin lesions, and can endanger tolerance and success of NIV.

One of the most unpleasant and painful iatrogenic interface-related complications in NIV is facial pressure ulcers. These are seen on areas of high pressure, mainly on the nasal bridge, but can develop at any place where the mask or headgear has contact with the skin (fig. 2). The reported occurrence rate in ARF varies depending on timing of the study, patient population, masks and skin protective strategies used. Reports from earlier NIV trials range from $5 \%$ to $56 \%$ [22, 23]; although improvements in mask design have helped to decrease the problem, more recent reports still range from $3 \%$ to $87 \%$ [24-27]. This can be partly explained by the expansion of NIV indications towards more severely ill patients at risk for pressure ulcers, ventilation with higher inspiratory pressures that promote air leaks, and the constantly increasing number of NIV services with initially less experienced staff and only a small selection of masks available. The fact that the respiratory support needed by the patient is naturally rated more of a priority by medical staff than the local skincare also contributes to the persistence of the problem.

Interface-related pressure ulcers are medical-device related pressure ulcers and the longer treatment lasts the more likely they are to develop. A number of patient-dependent risk factors facilitate their occurrence (table 2), but the literature shows that the main cause for these pressure ulcers is often associated with a lack of understanding of how and how often to remove devices and inspect the skin, and how to ensure the device fits appropriately to minimise friction and pressure [28]. Most of the patientdependent risk factors cannot be changed, but if mask fitting, regular assessment and skin protection are considered from the beginning of therapy and adapted to the individual patient's needs, skin breakdown can be avoided or at least reduced in many cases.

It is important to identify patients at risk of developing pressure ulcers from the interface so that preventive strategies can be initiated early. An initial assessment of every patient receiving NIV should include examination of skin integrity on the face and around the head, in areas where the skin comes into contact with the headgear, and the common risk factors for healthcare and device-related pressure ulcers. The skin should be reassessed at least once per nursing shift and ideally every time the mask is removed.

The most important strategy in the prevention of pressure ulcers is to keep the pressure applied to the skin as low as possible. Admittedly, it can be challenging to find the ideal strap tension and mask fit
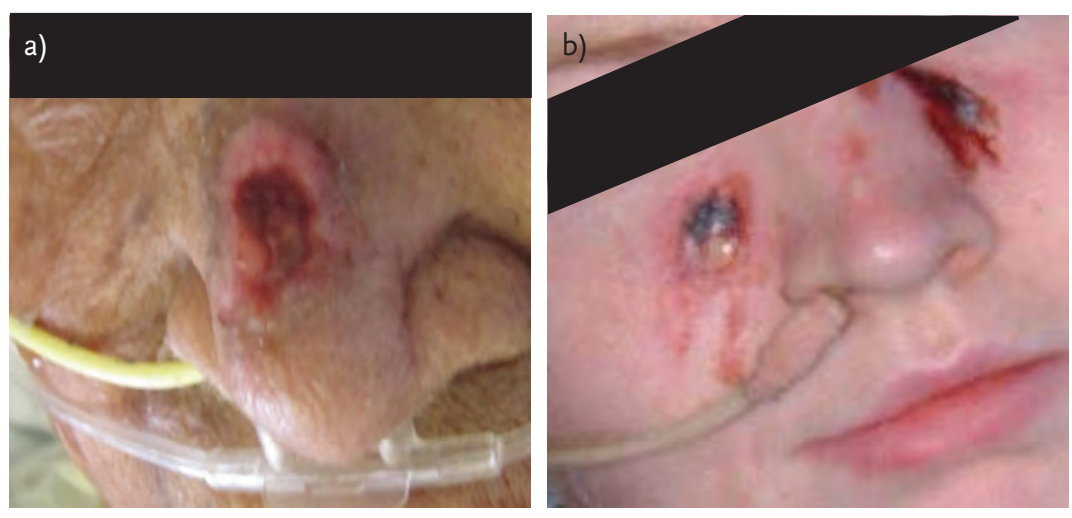

Figure 2

a) Grade III pressure ulcer on the nasal bridge and b) grade II pressure ulcers on the cheeks. 
Table 2 Risk factors for the development of interface-related pressure ulcers in NIV

\section{General risks}

Sensory impairment

Acute illness

Chronic illness

Hypoxia or very low blood pressure

Extremes of age

Low level of consciousness

Psychological status

Vascular disease

Malnutrition/dehydration

Chronic skin condition

History of previous pressure damage

Medication (e.g. analgesia, chronic steroid therapy, cytotoxins)

\section{Extrinsic factors}

Closely fitting headgear and over-tightened straps

Poorly fitted masks and headgear (i.e. too big or too small, too old or wrong style)

Mechanical forces: pressure, shear or friction from the interface

Allergy to the cushion

\section{Other factors}

Skin damage: dry, flaky, excoriated, discoloured or macerated skin

Oedema

Shape and size of nose/face

Time period that mask pressure is applied

Inability to self-manage the mask

that allows for both a good seal and low pressure on the skin. A physiological study on a head model showed that a pressure of the mask against the face about $2-3 \mathrm{cmH}_{2} \mathrm{O}$ higher than the peak inspiratory airway pressure is enough to prevent extensive air leaks [29]. Unfortunately, the pressure on the face cannot be measured objectively in everyday care or in every mask type and it does not give information on single points of high pressure, for example the nasal bridge. Therefore, the clinicians who fit the mask have to be guided by the patient's feedback and their experience. The correct size of mask and headgear with a higher number of attachment points will help to distribute the pressure more evenly around the circumference of the mask. Tightening the mask straps until the air leak is only just controlled, purposely leaving a very small air leak or to allow enough space to pass at least two fingers underneath the head strap (fig. 3) [30], can also help to avoid an over-tightening of the head straps.

Skin-protective strategies include ensuring the skin is clean and dry, as well as 


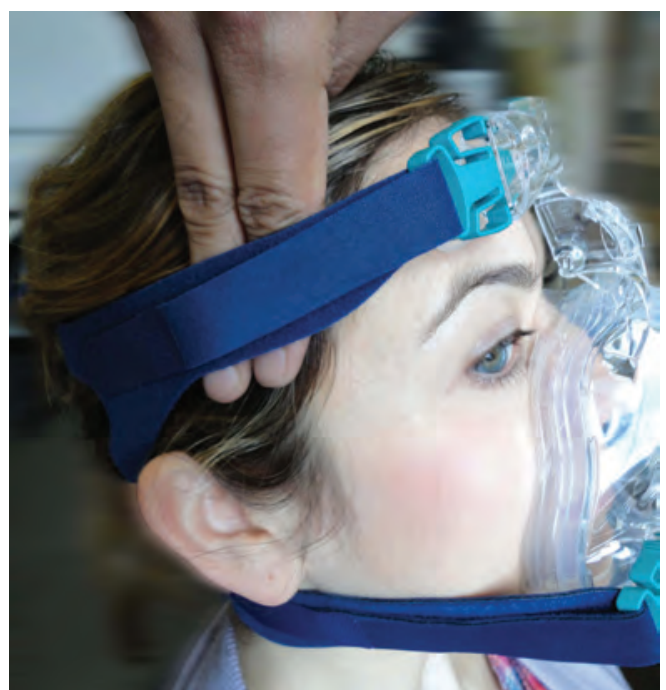

Figure 3

The two-finger rule: when the headgear is attached it should be possible to pass two fingers beneath it [30].

regular pressure relief, use of special mask cushions, and application of dressings to the skin to redistribute pressure and reduce friction. For patients with very prominent anatomy or at higher risk of developing pressure ulcers, masks with softer cushions (e.g. hydrogel cushions or double spring airfilled cushions, or a combination of both) can be used. An adjustable forehead spacer can also help to distribute the pressure more evenly on the face and reduce it on the bridge of the nose. For difficult cases, the choice of a more costly, but very comfortable mask, is appropriate and can be cost-effective if it avoids intubation.

Giving the skin regular breaks from interface pressure, ideally every $2-4 \mathrm{~h}$, is important to reduce pressure effects and allows for inspection of the skin underneath the mask. If this is not done, very severe skin and tissue damage can occur as demonstrated in a case report of a patient in whom the mask was not removed for $>90$ consecutive hours [31]. Some patients deteriorate rapidly when the mask is removed, but they can be changed to an alternative interface to relieve pressure on the tissue. This concept known as mask rotation, i.e. a planned sequential utilisation of different interfaces, can reduce pressure effects by alternating the points of highest pressure, and is also useful to improve the tolerance and efficacy of NIV [14]. The alternation of an oronasal mask and a full-face mask allows regular pressure relief of the nasal bridge. Other interfaces without contact with the nasal bridge, for example masks with a cut-out on the nasal bridge or nasal prongs, can also be tried. In patients at high risk of pressure ulcers, mask rotation should be started from the beginning.

The routine use of additional protective coverings applied to areas with the highest pressure can reduce the occurrence of pressure ulcers. Pads of foam [26], hydrocolloids [32] or gel distribute the pressure, reduce friction and can also reduce small air leaks at the same time (fig. 4 and 5). They are recommended for patients with a high risk of pressure ulcers from the beginning of NIV or, at the latest, from when the first signs of redness appear on the skin. It is important not to create more pressure on the skin when the dressing is placed (fig. 6).

The helmet is the only interface that does not create pressure ulcers on the face and is a useful alternative for experienced centres, if pressure effects on the face become a NIVlimiting problem. However, it has to be kept in mind that the use of a helmet can create skin damage along the braces under the armpits or around the neck. A case of arm vein thrombosis due to pressure from the attachment straps has been described in a very cachectic patient [33].

\section{Upper-airway obstruction}

High pressure from the lower mask strap can sometimes provoke or aggravate upper airway obstruction by pushing the lower jaw backwards. This is normally not a problem

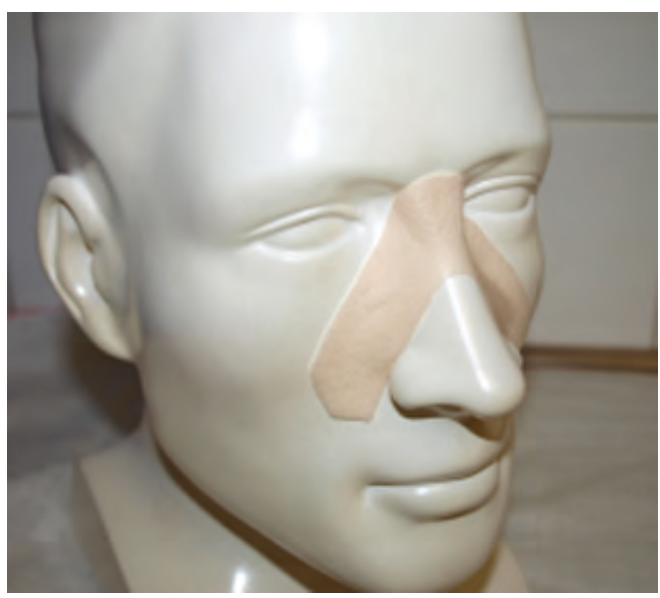

Figure 4

Example for skin protection: a self-cut foam (Mölnlycke Health Care, Göteborg, Sweden) dressing on a head model. 


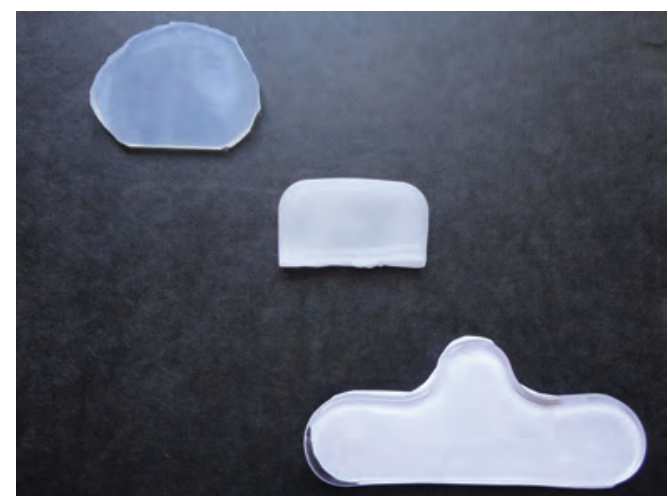

Figure 5

Different self-cut hydrocolloid and gel sheets to be placed between skin and mask. From left to right: Kerra Pro ${ }^{T M}$ (Crawford Healthcare Ltd, Knutsford, UK), Aderma (Smith a Nephew, London, UK) and Gecko $^{T M}$ (ResMed Ltd.)

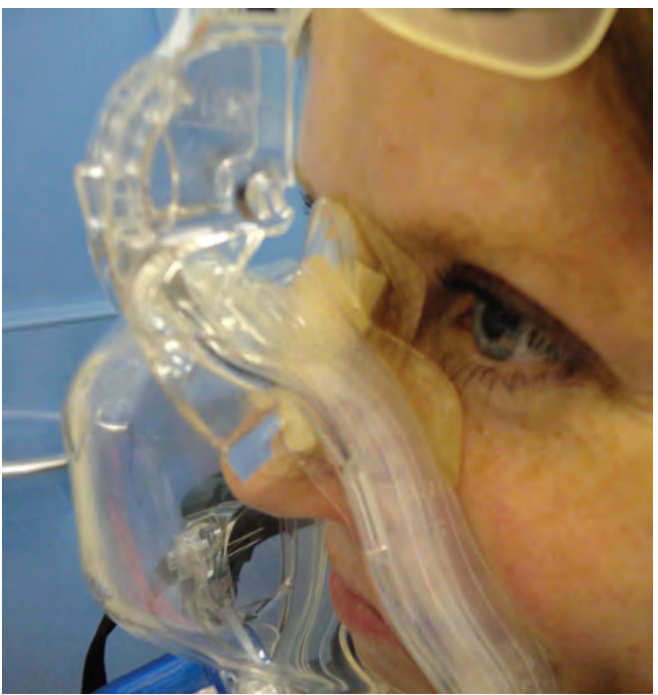

Figure 6

Foam and silicone sheets between mask and skin.

when the mask is initially fitted in an upright or semi-recumbent position, but might be seen in some patients in the supine position or during rapid eye movement sleep. The obvious solution to this problem is to loosen the straps, refit the mask or slightly increase the expiratory positive airway pressure. When a secure mask fit cannot be obtained without higher strap tension, the addition of a stiff neck collar or a rolled-up towel under the chin can be considered to help keep the upper airway open.

\section{Skin}

To reduce the risk of skin irritation it is important to keep the skin and mask clean and dry throughout the treatment. If redness, rashes or blistering occur, a change to a silicone and latex free mask cushion, application of barrier cream and covering the damaged skin to protect it when the mask is in place can all be tried. If an allergic rash occurs, the mask cushion should be changed to a different material and application of steroid cream can be considered.

\section{Regular reassessment}

Movements, sweating, change of fluid status and the tendency of Velcro straps to loosen gradually over time can change mask fit. Thus, no matter how well the interface fits at the start of NIV, it is important to routinely monitor air leaks and skin condition and to reassess mask fit throughout the treatment.

\section{Dryness of mucosa and humidification}

Oral and nasal dryness or a blocked nose are frequent complaints during NIV with an occurrence rate of $10-50 \%[1,2,34]$. They are indicative of air leaking through the mouth or around the mask, but even without an air leak, patients can be troubled by these symptoms. Cold, dry air from the ventilator, often caused by a higher oxygen fraction, promotes the release of inflammatory mediators in the nasal mucosa, increases mucosal blood flow and thereby increases nasal airway resistance and causes congestion. Treatment options include the topical nasal application of saline, hyaluronic acid, steroids, decongestants or antihistamines, and regular mouth care, as well as the addition of heat/moisture exchangers and an external heated humidifier to the circuit.

The use of active humidification of the inspired gas is mandatory for patients with a tracheostomy, but its use in NIV is not always necessary. Nevertheless, it should be added to the circuit with a low threshold, especially if leaks are present or a high oxygen fraction is delivered as it can attenuate the increase in resistance in the nasal mucosa, decrease dryness and enhance comfort and tolerance of mask ventilation [35]. It is also recommended to avoid thickened and tenacious secretions. Gas temperatures during NIV do not need to be as great as for intubated patients and are to be based on patient comfort (benchmark of 100\% relative humidity at about $30^{\circ} \mathrm{C}$ ) [36]. Heat/moisture exchangers 
cannot be unconditionally recommended for NIV, because the additional dead space can decrease $\mathrm{CO}_{2}$ elimination and increase the work of breathing, which can become problematic, in particular in hypercapnic ARF or if the delivered tidal volumes are very low [37].

\section{Sedation}

The topic of sedative medication to improve tolerance of the NIV interface is still controversial. The idea is to mitigate the patient's level of discomfort and obtain a level of awake sedation without significant effects on respiratory drive, minute volume, respiratory pattern, blood gases, haemodynamics and airway reflexes. The best way to improve interface tolerance during NIV is still good nursing and multidisciplinary care with intensive psychological support and reassurance of the patient. However, for some patients who remain very anxious or agitated with the mask, a small dose of an anxiolytic may be appropriate. Many pulmonary and critical care physicians in North America and Europe are reluctant to use sedation because of the perceived risks of interfering with the ability to protect the airway or depressing respiratory drive [38]. Nevertheless, careful and mild sedation in selected patients in ICUs with a high nurse to patient ratio and special focus and attention on airway protection and consciousness is usually safe, and can sometimes help to prevent NIV failure due to low tolerance. The ability to use sedatives or analgesics on wards with a lower degree of monitoring is very limited and cannot be recommended.

\section{Acute-on-chronic respiratory failure}

The number of patients with chronic respiratory failure who are on long-term home NIV is also constantly on the increase. These patients are normally treated in specialised centres, but during acute exacerbations may present to the nearest emergency department or ICU for initial treatment. They will often bring their own mask and ventilator equipment with them and, while it is usually necessary to adjust the ventilator settings during the exacerbation, their mask can continue to be used. Although these patients are "expert patients" concerning their mask management and NIV, this does not imply that they will not have mask problems in the acute setting. Patients who are well adapted to nasal masks or nasal prongs at home might suddenly start mouth breathing during the exacerbation, which will necessitate a temporary switch to an oronasal mask or total facemask. The risk for facial pressure ulcers can increase in the context of a more severe illness with, for example, worse oxygenation or lower blood pressure. Increases in pressure support can lead to air leaks and consequently increased tightening of the straps might follow. In addition, patients may suddenly find themselves on NIV for most of the 24 -h period instead of only using it at night, or be unable to correctly selfmanage the mask because of weakness. These patients will have to be able to continue the treatment after the exacerbation, which can become very uncomfortable or painful with a pressure sore on the face and prolonged wound healing due to complicated pressure relief. Patients on home NIV can have the same mask problems as all other patients in the acute setting and there is a need for regular reassessment of mask fit, skin underneath the mask and headgear, risk factors for pressure ulcers and for precautions to reduce the incidence of skin damage or NIV failure.

\section{Feeding tubes, beards and other problems}

NIV can be used with a nasogastric tube in place and some masks incorporate a special nasogastric tube adapter. If such a mask or a separate nasogastric tube adapter is not available, padding of the tube and the skin underneath the tube with a silicone dressing or other material is recommended to minimise air leakage and pressure on the skin. Regular reassessment of the skin under the nasogastric tube, in particular in the area where it is in contact with the mask frame, should be carried out and the position of the tube alternated if the treatment has to be prolonged.

Another frequent problem mainly associated with air leaks is beards. Shaving is a very quick and effective solution to this problem, but if this is inappropriate, an oronasal mask is usually more effective with 
moustaches than nasal masks, and full beards can sometimes be managed better with nasal prongs, total face masks, or with helmets in the ICU of an experienced centre.

Dentures can also become a problem, as a mask that fits very well with the denture in place might not fit once the denture is removed at night. It is important to be aware of this potential change in mask fit and to have an alternative mask quickly available, if needed.

For patients who are unable to raise their arms to pull the mask over their head or to reach the strap to remove the mask themselves because of weakness, neuromuscular disease or severe arthritis, a quick-release strap can be added.

\section{Droplet generation and airborne disease}

NIV is a droplet-generating procedure, which raises concerns about infection control and staff protection with its use during outbreaks of diseases, such as pandemic influenza $\left(\mathrm{H}_{1} \mathrm{~N}_{1}\right)$ or severe acute respiratory syndrome, or with airborne diseases such as tuberculosis. The amount of droplets falls significantly at a distance $>_{1} \mathrm{~m}$ from the patient $[39,40]$, but healthcare workers who apply NIV have to stand close to the patient and, therefore, can be exposed to potentially infectious droplets. The decision whether or not NIV can be used in these situations, as well as the general precautions to reduce disease transmission, usually depends on local infection policies. However, if NIV is applied, a nonvented facial mask and filtered exhalate can reduce environmental spread [39]. Also, fitting and securing the mask before the ventilator is turned on and similarly turning off the ventilator before the mask is removed or lifted away from the face is usually recommended.

\section{Conclusion}

Skills in mask fitting and prevention of maskrelated problems are key components for NIV success. Not all hospitals and NIV services will be able to stock a large volume of different masks, which makes routine risk assessment for mask-related pressure ulcers, and knowledge and strategic use of skin protective strategies even more important. Training programmes for NIV should impart this knowledge and qualify the team to tailor interface choice and preventive strategies to each patient's individual facial characteristics and needs.

\section{Acknowledgements}

The author is the recipient of a European Respiratory Society Fellowship (LTRF 20131798) funded jointly by the European Respiratory Society and the European Lung Foundation. The author wishes to thank Anita Simonds (Dept of Sleep and Ventilation, Royal Brompton and Harefield NHS Foundation Trust, London, UK) for helpful advice and critical review of the manuscript and Manuela Del Forno (Respiratory and Critical Care Unit, University of Bologna, Bologna, Italy) for her assistance with the pictures.

\section{Educational questions}

1) Which of the following statements concerning mask choice is/are true?

a. Masks covering mouth and nose are more effective in improving blood gases than nasal masks in acute respiratory failure

b. Oronasal masks are better tolerated and more comfortable than nasal masks in acute respiratory failure

c. Oronasal masks are usually the first choice for NIV in acute respiratory failure in Europe

d. Mask switching is not recommended in the first $24 \mathrm{~h}$ of NIV in acute respiratory failure

2) Which of the following statements concerning interface-related pressure ulcers in NIV is/ are true?

a. Pressure ulcers occur more often with oronasal than nasal masks

b. A helmet cannot create pressure ulcers

c. The use of skin protective dressings can reduce the incidence of pressure ulcers 
d. The most important strategy to prevent mask-related pressure ulcers is rotation of different masks

3) A 44-year-old woman, who is on home NIV because of an obesity hypoventilation syndrome, presents to the emergency department with a chest infection and exacerbation of her chronic respiratory failure. She has brought her ventilator and mask with her and is started on this with an increased inspiratory positve airway pressure (IPAP) and the nasal mask that she uses at home. At the higher IPAP, the patient develops a mouth air leak. She refuses to change the mask to an oronasal or total face mask because of claustrophobia.

Which of the following can be expected with a persistent mouth air leak?

a. The expiratory volume increases

b. The intra-alveolar pressure is not influenced

c. A further increase in pressure support can partly compensate for the leak

d. Nasal airway resistance decreases.

e. Heated humidification does not have an influence on nasal resistance in this situation

4) A 58-year-old smoker presents with an exacerbation of his chronic obstructive pulmonary disease to the emergency department. He is tachypnoeic (respiratory rate 32 per min) and the initial arterial blood gas analysis on room air shows $\mathrm{pH} 7.28$, arteral oxygen tension $\left(\mathrm{PaO}_{2}\right)$ $50 \mathrm{mmHg}$ and arterial carbon dioxide tension $\left(\mathrm{PaCO}_{2}\right) 58 \mathrm{mmHg}$. He is started on nebulised bronchodilators, steroids and antibiotics, and NIV is added with a facial mask and a ventilator in the emergency department. He synchronises well with the ventilator, his respiratory rate decreases to 23 per min after $1 \mathrm{~h}$, blood gases improve to $\mathrm{pH} 7.32, \mathrm{PaO}_{2}$ $64 \mathrm{mmHg}$ and $\mathrm{PaCO}_{2} 50 \mathrm{mmHg}$, and the patient feels better. He is transferred to a HDU and NIV is restarted with a smaller ventilator. The patient is awake, feels better and is still doing well on the ventilator. There is no significant air leak, no signs of auto-positive end-expiratory pressure, patient ventilator asynchrony or pneumothorax, but the next arterial blood gas shows a pH of 7.29, $\mathrm{PaO}_{2} 60 \mathrm{mmHg}$ and $\mathrm{PaCO}_{2}$ of $65 \mathrm{mmHg}$. What is the most appropriate next step?

a. Continue the patient on oxygen only

b. Repeat the arterial blood gas analysis

c. Check the equipment used (ventilator, circuit and mask)

d. Intubate the patient immediately

e. Change to a nasal mask

References

1. Nava S, Hill N. Non-invasive ventilation in acute respiratory failure. Lancet 2009; 374: 250-259.

2. Mehta S, Hill NS. Noninvasive ventilation. Am J Respir Crit Care Med 2001; 163: 540-577.

3. Plant PK, Owen JL, Elliott MW. Early use of noninvasive ventilation for acute exacerbations of chronic obstructive pulmonary disease on general respiratory wards: a multicentre randomised controlled trial. Lancet 2000; 355: 1931-1935.

4. Poponick JM, Renston JP, Bennett RP, et al. Use of a ventilatory support system (BiPAP) for acute respiratory failure in the emergency department. Chest 1999; 116: 166-171.

5. Schettino G, Altobelli N, Kacmarek RM. Noninvasive positive-pressure ventilation in acute respiratory failure outside clinical trials: experience at the Massachusetts General Hospital. Crit Care Med 2008; 36: 441-447.

6. Thille AW, Contou D, Fragnoli C, et al. Non-invasive ventilation for acute hypoxemic respiratory failure: intubation rate and risk factors. Crit Care 2013; 17: R269.
7. Cabrini L, Idone C, Colombo S, et al. Medical emergency team and non-invasive ventilation outside ICU for acute respiratory failure. Intens Care Med 2009; 35: 339-343.

8. Soo Hoo GW, Santiago S, Williams AJ. Nasal mechanical ventilation for hypercapnic respiratory failure in chronic obstructive pulmonary disease: determinants of success and failure. Crit Care Med 1994; 22: 1253-1261.

9. Hill NS. Complications of noninvasive ventilation. Respir Care 2000; 45: 480-481.

10. Carron M, Freo U, BaHammam AS, et al. Complications of non-invasive ventilation techniques: a comprehensive qualitative review of randomized trials. Br J Anaesth 2013; 110: 896-914.

11. Kwok H, McCormack J, Cece R, et al. Controlled trial of oronasal versus nasal mask ventilation in the treatment of acute respiratory failure. Crit Care Med 2003; 31: 468-473.

12. Girault C, Briel A, Benichou J, et al. Interface strategy during noninvasive positive pressure ventilation for 


\section{Suggested answers}

1) a. false, b. false, c. true, d. false

2) a. true, b. false, c. true, d. false

3) $c$.

4) c. hypercapnic acute respiratory failure. Crit Care Med 2009; 37: 124-131.

13. Crimi C, Noto A, Princi P, et al. A European survey of noninvasive ventilation practices. Eur Respir J 2010; 36: $362-369$.

14. Navalesi $\mathrm{P}$, Fanfulla F, Frigerio $\mathrm{P}$, et al. Physiologic evaluation of noninvasive mechanical ventilation delivered with three types of masks in patients with chronic hypercapnic respiratory failure. Crit Care Med 2000; 28: 1785-1790.

15. Schettino GP, Chatmongkolchart S, Hess DR, et al. Position of exhalation port and mask design affect $\mathrm{CO}_{2}$ rebreathing during noninvasive positive pressure ventilation. Crit Care Med 2003; 31: 2178-2182.

16. Fraticelli AT, Lellouche F, L'Her E, et al. Physiological effects of different interfaces during noninvasive ventilation for acute respiratory failure. Crit Care Med 2009; 37: 939-945.

17. Carlucci A, Richard JC, Wysocki M, et al. Noninvasive versus conventional mechanical ventilation. An epidemiologic survey. Am J Respir Crit Care Med 2001; 163: 874-880.

18. Olivieri C, Costa R, Conti G, et al. Bench studies evaluating devices for non-invasive ventilation: critical analysis and future perspectives. Intensive Care Med 2012; 38: 160-167.

19. Bachour A, Hurmerinta K, Maasilta P. Mouth closing device (chinstrap) reduces mouth leak during nasal CPAP. Sleep Med 2004; 5: 261-267.

20. Teschler H, Stampa J, Ragette R, et al. Effect of mouth leak on effectiveness of nasal bilevel ventilatory assistance and sleep architecture. Eur Respir J 1999; 14: 1251-1257.

21. Storre JH, Bohm P, Dreher M, et al. Clinical impact of leak compensation during non-invasive ventilation. Respir Med 2009; 103: 1477-1483.

22. Ambrosino N. Noninvasive mechanical ventilation in acute respiratory failure. Eur Respir J 1996; 9: 795-807.

23. Nava $\mathrm{S}$, Ambrosino N, Clini E, et al. Noninvasive mechanical ventilation in the weaning of patients with respiratory failure due to chronic obstructive pulmonary disease. A randomized, controlled trial. Ann Intern Med 1998; 128: 721-728.

24. Fischer C, Bertelle V, Hohlfeld ), et al. Nasal trauma due to continuous positive airway pressure in neonates. Arch Dis Child Fetal Neonatal Ed 2010; 95: F447-F451.

25. Lemyze M, Mallat J, Nigeon O, et al. Rescue therapy by switching to total face mask after failure of face mask-delivered noninvasive ventilation in do-notintubate patients in acute respiratory failure. Crit Care Med 2013; 41: 481-488.

26. Weng $\mathrm{MH}$. The effect of protective treatment in reducing pressure ulcers for non-invasive ventilation patients. Intensive Critical Care Nurs 2008; 24: 295-299.
27. Gregoretti C, Confalonieri M, Navalesi P, et al. Evaluation of patient skin breakdown and comfort with a new face mask for non-invasive ventilation: a multi-center study. Intensive Care Med 2002; 28: 278-284.

28. Apold J, Rydrych D. Preventing device-related pressure ulcers: using data to guide statewide change. J Nurs Care Qual 2012; 27: 28-34.

29. Schettino GP, Tucci MR, Sousa R, et al. Mask mechanics and leak dynamics during noninvasive pressure support ventilation: a bench study. Intensive Care Med 2001; 27: 1887-1891.

30. Meduri GU, Abou-Shala N, Fox RC, et al. Noninvasive face mask mechanical ventilation in patients with acute hypercapnic respiratory failure. Chest 1991; 100: 445-454.

31. Ahmad Z, Venus M, Kisku W, et al. A case series of skin necrosis following use of non invasive ventilation pressure masks. Int Wound J 2013; 10: 87-90.

32. Callaghan S, Trapp M. Evaluating two dressings for the prevention of nasal bridge pressure sores. Prof Nurse 1998; 13: 361-364.

33. Antonelli M, Pennisi MA, Pelosi P, et al. Noninvasive positive pressure ventilation using a helmet in patients with acute exacerbation of chronic obstructive pulmonary disease: a feasibility study. Anesthesiology 2004; 100: 16-24.

34. Lellouche F, Maggiore SM, Lyazidi A, et al. Water content of delivered gases during non-invasive ventilation in healthy subjects. Intensive Care Med 2009; 35: 987-995.

35. Tuggey JM, Delmastro M, Elliott MW. The effect of mouth leak and humidification during nasal noninvasive ventilation. Respir Med 2007; 101: 1874-1879.

36. Esquinas Rodriguez AM, Scala R, Soroksky A, et al. Clinical review: humidifiers during non-invasive ventilation - key topics and practical implications. Crit Care 2012; 16: 203.

37. Lellouche F, Pignataro C, Maggiore SM, et al. Shortterm effects of humidification devices on respiratory pattern and arterial blood gases during noninvasive ventilation. Respir Care 2012; 57: 1879-1886.

38. Devlin JW, Nava S, Fong JI, et al. Survey of sedation practices during noninvasive positive-pressure ventilation to treat acute respiratory failure. Crit Care Med 2007; 35: 2298-2302.

39. Simonds AK, Hanak A, Chatwin M, et al. Evaluation of droplet dispersion during non-invasive ventilation, oxygen therapy, nebuliser treatment and chest physiotherapy in clinical practice: implications for management of pandemic influenza and other airborne infections. Health Technol Assess 2010; 14: 131-172.

40. Hui DS, Chow BK, Ng SS, et al. Exhaled air dispersion distances during noninvasive ventilation via different Respironics face masks. Chest 2009; 136: 998-1005. 